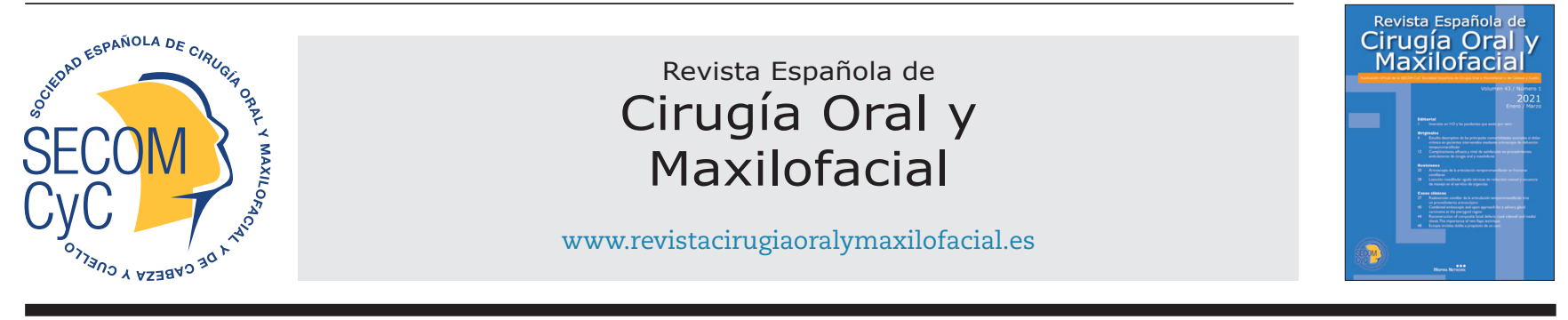

\title{
Revisión
}

\section{Luxación mandibular aguda: técnicas de reducción manual y secuencia de manejo en el servicio de urgencias}

\author{
Paula Astorga Jélvez ${ }^{1,2^{*}}$, Marcela Garrido ${ }^{3}$ y Emilio Moreno Apablaza ${ }^{1,4}$
}

${ }^{1}$ Cirugía Maxilofacial, Universidad de los Andes. Santiago, Chile. ${ }^{2}$ Servicio de Urgencia, Clínica UC San Carlos de Apoquindo. Santiago, Chile. ${ }^{3}$ Servicio de Urgencia, Departamento de Salud Pública y Epidemiología, Clínica Universidad de los Andes. Santiago, Chile. ${ }^{4}$ Servicio de Urgencia, Hospital de Carabineros del General Humberto Arriagada Valdivieso. Santiago, Chile

\section{INFORMACIÓN DEL ARTÍCULO}

Historia del artículo:

Recibido: 4 de julio de 2020

Aceptado: 29 de octubre de 2020

Palabras clave:

Luxación de la articulación temporomandibular, luxación

de la ATM, luxación mandibular.

\section{R E S U M E N}

Introducción: El objetivo del presente trabajo es realizar una revisión actualizada sobre los métodos de reducción manual de la luxación mandibular aguda para proponer una secuencia de manejo en el servicio de urgencia.

Material y métodos: La revisión incluyó estudios sobre técnicas manuales de reducción de luxaciones mandibulares agudas. La búsqueda se realizó en inglés y español en junio de 2020 e incluyó las bases de datos electrónicas PubMed y Scielo, complementada por referencias cruzadas. Se excluyeron artículos sobre tratamiento quirúrgico de luxaciones recidivantes, crónicas y luxaciones reducidas bajo anestesia general.

Resultados: La búsqueda arrojó 334 resultados, finalmente 29 estudios fueron incluidos. A la fecha hay descritas 13 técnicas de reducción manual distintas al método convencional. El método convencional tiene una efectividad cercana al $86 \%$, el método de pivote de muñeca $96,7 \%$ y el método extraoral es menos efectivo con un porcentaje de éxito entre 55,2 y $66,7 \%$. Se propone una secuencia de tratamiento incluyendo los aspectos más relevantes encontrados en la literatura.

Conclusión: La mejor técnica de reducción manual es aquella que entrega mayor comodidad al profesional, produce menor dolor y se asocia a una mayor tasa de éxito. Faltan estudios que comparen distintas técnicas con un operador entrenado considerando variables del paciente, de la técnica y la comodidad para el operador. Es fundamental conocer las técnicas presentadas; esto permite utilizarlas en los casos donde otras técnicas fracasen, minimizando el uso de coadyuvantes y maniobras más complejas, como bloqueos regionales o anestesia general.

\footnotetext{
${ }^{*}$ Autor para correspondencia:

Correo electrónico: paula.astorgaj@gmail.com (Paula Astorga Jélvez). 


\section{Acute mandibular luxation: manual reduction techniques and management sequence in the emergency department}

\section{A B S T R A C T}

Keywords:

Temporomandibular joint luxation, TMJ luxation, mandibular luxation.
Introduction: The objective of the present study is to carry out an updated review on the methods of manual reduction of acute mandibular dislocation to propose a management sequence in the emergency department.

Material and methods: The review included studies on manual techniques for reducing acute mandibular dislocations. The search was performed in Spanish and English in june 2020 and included the electronic databases PubMed and Scielo, complemented by cross-references. Articles on surgical treatment of recurrent, chronic and reduced dislocations under general anesthesia were excluded.

Results: The search yielded 334 results, finally 29 studies were included. To date, 13 manual reduction techniques different from the conventional method have been described. The effectiveness of the conventional method is close to $86 \%$, the wrist pivot method $96.7 \%$ and the extraoral method is less effective with a success rate between 55.2 and $66.7 \%$. A treatment sequence is proposed including the most relevant aspects found in the literature.

Conclusion: The best manual reduction technique is one that provides greater comfort to the professional, produces less pain and is associated with a higher success rate. Studies comparing different techniques with a trained operator are lacking considering patient, technique and operator comfort variables. It is essential to know the techniques presented, this allows them to be used in cases where other techniques fail, minimizing the use of adjuvants and more complex maneuvers, such as regional blocks or general anesthesia.

\section{INTRODUCCIÓN}

La luxación mandibular ocurre cuando el cóndilo mandibular se desplaza anteriormente más allá de la eminencia articular y la cabeza condilar se ubica completamente fuera de la cavidad glenoidea ${ }^{1}$. Esto puede ocurrir secundario a un trauma o espontáneamente ${ }^{1,2}$. El tratamiento está dirigido a guiar la cabeza del cóndilo hacia atrás y hacia abajo a lo largo de la eminencia articular del hueso temporal de regreso a la fosa glenoidea ${ }^{3}$.

Tradicionalmente el diagnóstico es clínico y para el estudio imagenológico se han utilizado radiografías convencionales y tomografías computarizadas. La imagen a menudo es innecesaria en el contexto de una presentación clínica indicativa de luxación mandibular, sin antecedentes de trauma agudo ${ }^{1,4,5}$.

Se estima que la luxación mandibular corresponde al $3 \%$ de las luxaciones articulares del cuerpo $0^{6}$. En un servicio de urgencia con aproximadamente 100.000 visitas anuales, se presentaron 37 casos de luxación mandibular durante un periodo de 7 años ${ }^{7}$.

La técnica de reducción convencional, conocida como maniobra de Nelaton, se encuentra descrita en el papiro de

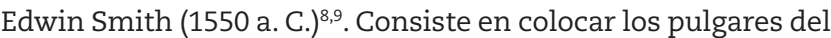
operador sobre los molares inferiores del paciente y empujar la mandíbula dislocada hacia abajo y hacia atrás. Tradicionalmente el paciente se encuentra sentado y el operador se posiciona de pie frente al paciente. Se ha descrito que la reducción manual utilizando la técnica convencional conlleva el riesgo de mordedura accidental de los dedos y, con ello, transmisión de enfermedades. Por esto se han descrito nuevas formas de lograr reposicionar los cóndilos mandibulares dentro de la fosa articular.
El objetivo del presente trabajo es revisar los métodos de reducción manual de la luxación mandibular aguda para proponer una secuencia de manejo en el servicio de urgencia.

\section{MATERIALES Y MÉTODOS}

\section{Desarrollo de un protocolo y pregunta enfocada}

El método utilizado en esta revisión se adaptó de los elementos de informe preferidos para revisiones sistemáticas y metanálisis (PRISMA) y la guía preparada por Needleman. Las preguntas clínicas se formularon y organizaron de acuerdo con el marco PICO para práctica basada en la evidencia.

La pregunta para definir la estrategia de búsqueda fue: ¿cuál es la mejor técnica manual para reducir la luxación mandibular aguda?

\section{Estrategia de búsqueda}

La estrategia de búsqueda se basó en las directrices de PRISMA, desde enero de 1980 hasta junio de 2020. Se realizó una búsqueda electrónica exhaustiva en las bases de datos MEDLINE/PubMed (en inglés) y en Scielo (inglés y español), para publicaciones relevantes en revistas indexadas. Como filtros de búsqueda se limitó sin restrictor por idioma y se utilizaron las siguientes palabras claves: "Temporomandibular joint luxation", "TMJ luxation”, “Temporomandibular joint dislocation”, "TMJ dislocation", "Mandibular luxation", "Mandibular dislocation", "Acute”, "Reduction", “Treatment". 


\section{Revisión y selección}

Se incluyeron notas técnicas, revisiones narrativas, reportes y series de casos, estudios descriptivos, ensayos clínicos y revisiones sistemáticas, en humanos, que abordaran técnicas manuales de reducción aguda de luxaciones mandibulares.

Los dos revisores realizaron la detección de títulos y resúmenes para su posible inclusión de forma independiente.

Los estudios completos seleccionados se leyeron cuidadosamente y se analizaron para los criterios de elegibilidad y la extracción de datos. Las diferencias entre los revisores se resolvieron mediante discusión y consenso. Se usaron los siguientes criterios de exclusión: artículos sobre tratamiento quirúrgico de luxaciones recidivantes, luxaciones en el contexto de uso de anestesia general, luxaciones reducidas bajo anestesia general, tratamiento de luxaciones crónicas.

\section{RESULTADOS}

La búsqueda inicial arrojó 334 resultados. Se incluyeron 29 artículos según los criterios ya descritos. Debido a las diferencias metodológicas entre los estudios, se realizó una exposición de datos como resumen de hallazgos distribuidos en la Tabla I. Se describen 13 técnicas de reducción manual además del método convencional.

\section{Procedimientos de reducción manual}

En la literatura se describen técnicas que se asemejan a la convencional, pero incluyen variaciones que buscan aumentar la comodidad para el operador. Stakesby Lewis ${ }^{10}$ sugiere intentar inicialmente la reducción de un lado en luxaciones bilaterales. También sugiere ubicar al paciente en posición supina para realizar la maniobra de reducción desde atrás, con el mismo principio de aplicar una fuerza hacia abajo en el borde anterior de la rama y una fuerza hacia arriba en el mentón (Figura 1). Varios autores ${ }^{12,18,21,27}$ describen la ventaja de esta modificación. Cheng y cols. ${ }^{16}$ utilizan el mismo principio de fuerza hacia caudal y dorsal apoyando ambos pulgares unilateralmente en un sector posterior mandibular para ejercer una mayor fuerza de reducción que la lograda con el método convencional. En la técnica descrita por Stolbizer y cols. ${ }^{31}$ el énfasis está en la presión sostenida con sus pulgares en la zona retromolar en 45 grados hacia caudal; esto se acompaña en forma simultánea de movimientos mandibulares realizados por el paciente para lograr la reducción.

Otra técnica descrita es la reportada por Lowery y cols. ${ }^{7}$, el método de "pivote de muñeca". En este reporte de caso utilizan esta técnica después de dos intentos fallidos con la técnica convencional asociada a sedación con $3 \mathrm{mg}$ de midazolam y $50 \mu \mathrm{g}$ de fentanilo. La técnica consiste en ponerse frente al paciente de pie mientras la mandíbula se sujeta con los pulgares del operador en la punta del mentón y los dedos sobre la superficie oclusal de los molares inferiores. Al aplicar una fuerza cefálica con los pulgares y presionar hacia caudal con los dedos, girando las muñecas, la mandíbula dislocada se reduce sin dificultad (Figura 2). La ventaja que describen los autores es que en lugar de intentar alargar los músculos que están en espasmo, utiliza

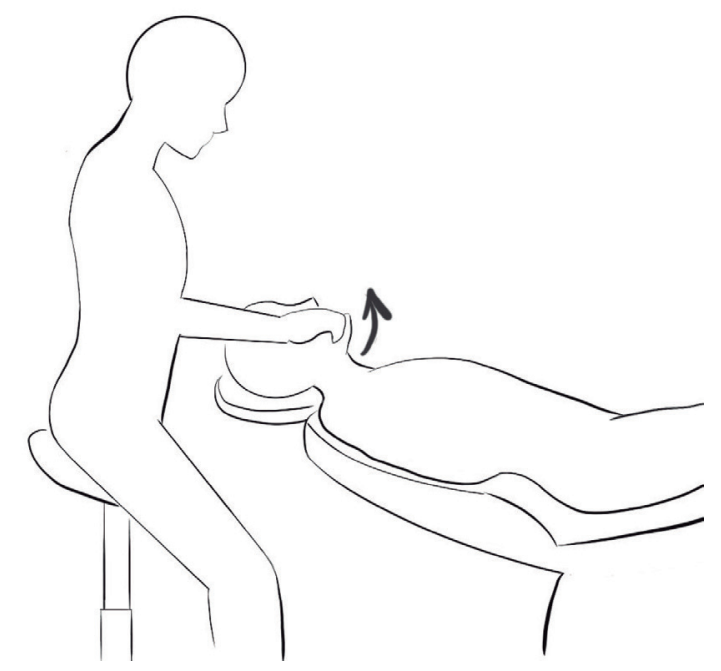

Figura 1. Técnica de posición supina: en esta técnica el operador se posiciona detrás del paciente, apoya ambos pulgares en el borde anterior de la rama, distal a los últimos molares mandibulares, y realiza un movimiento de rotación empujando el ángulo mandibular hacia caudal y el mentón hacia cefálico.

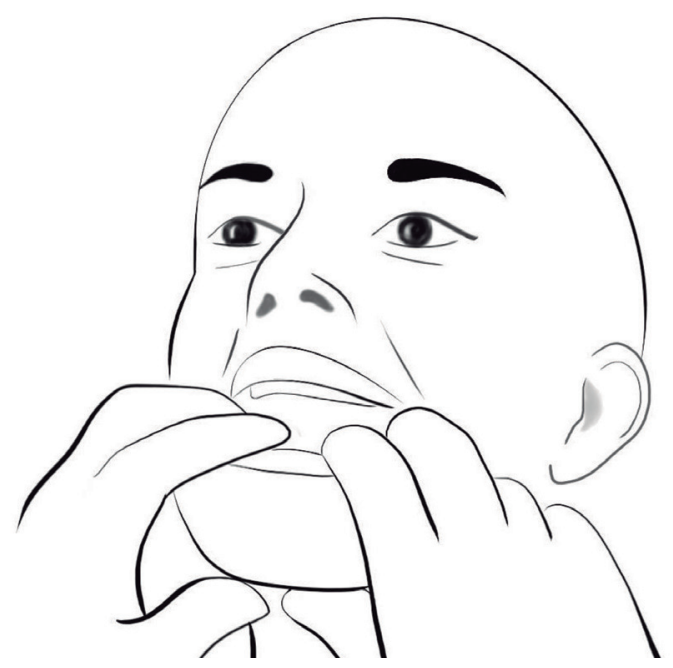

Figura 2. Técnica "pivote de muñeca": el operador se posiciona frente al paciente, apoya sus dedos índice y medio en el sector mandibular posterior, los pulgares en el borde inferior del mentón y luego realiza un movimiento de rotación empujando el mentón hacia cefálico.

la acción muscular en conjunto con las fuerzas aplicadas por el operador, para una reducción más suave y cómoda para el paciente y el operador. Lum $^{30}$ reporta un caso en donde utiliza esta técnica exitosamente, después de varios intentos fallidos con el método convencional incluso bajo sedación. Este caso resalta la necesidad de considerar métodos alternativos de reducción, particularmente en casos refractarios. 
Tabla I. Estudios incluidos en el análisis

\begin{tabular}{|c|c|c|c|c|}
\hline Autor & Tipo de estudio & Técnica descrita & & Tasa de éxito \\
\hline $\begin{array}{l}\text { Stakesby } \\
\text { Lewis, } 1981^{10}\end{array}$ & Serie de casos & $\begin{array}{l}\text { Convencional unilateral, "Recumbent approach" } \\
\text { (posición supina) }\end{array}$ & $\mathrm{n}=8$ & \\
\hline Awang, $1987^{11}$ & Serie de casos & Reflejo nauseoso & $\mathrm{n}=3$ & \\
\hline $\begin{array}{l}\text { Luyk y Larsen, } \\
1989^{12}\end{array}$ & Revisión narrativa & $\begin{array}{l}\text { Convencional, "Recumbent approach" (posición } \\
\text { supina) }\end{array}$ & & \\
\hline $\begin{array}{l}\text { Lowery y cols., } \\
200^{4} 7\end{array}$ & Reporte de caso & Pivote de muñeca & $\mathrm{n}=1$ & \\
\hline $\begin{array}{l}\text { Shun y cols., } \\
2006^{13}\end{array}$ & Cohorte prospectiva & Approach ipsilateral & $\mathrm{n}=24$ & $\begin{array}{c}\text { Método extraoral = } 54 \% \\
(13 / 24) \\
\text { Método intraoral }=90,9 \% \\
(10 / 11)\end{array}$ \\
\hline $\begin{array}{l}\text { Chen y cols., } \\
2007^{3}\end{array}$ & Serie de casos & Método extraoral & $\mathrm{n}=7$ & \\
\hline $\begin{array}{l}\text { Oliphant y cols., } \\
2008^{14}\end{array}$ & Reporte de caso & Convencional & $\mathrm{n}=1$ & \\
\hline $\begin{array}{l}\text { Chan y cols., } \\
2008^{1}\end{array}$ & Revisión narrativa & $\begin{array}{l}\text { Convencional, recumbent approach (posición } \\
\text { supina), posterior approach (posición supina), } \\
\text { approach ipsilateral, "método manual alternativo" } \\
\text { (similar a método extraoral del approach } \\
\text { ipsilateral), pivote de muñeca, reflejo nauseoso }\end{array}$ & & \\
\hline \multirow{2}{*}{$\begin{array}{l}\text { Ardehali y cols., } \\
2009^{15}\end{array}$} & \multirow{2}{*}{$\begin{array}{l}\text { Ensayo clínico } \\
\text { aleatorizado }\end{array}$} & Método extraoral & $\mathrm{n}=29$ & $55,2 \%(n=16)$ \\
\hline & & Método convencional & $\mathrm{n}=29$ & $86,2 \%(n=25)$ \\
\hline Cheng, $2010^{16}$ & Reporte de caso & Manos unidas & $\mathrm{n}=4$ & \\
\hline $\begin{array}{l}\text { Akinbami y cols., } \\
2011^{2}\end{array}$ & Revisión narrativa & $\begin{array}{l}\text { Convencional, pivote de muñeca, approach } \\
\text { ipsilateral, método extraoral, reflejo nauseoso }\end{array}$ & & \\
\hline $\begin{array}{l}\text { Osawa y cols., } \\
2011^{17}\end{array}$ & Revisión narrativa & $\begin{array}{l}\text { Convencional, manos unidas, método extraoral, } \\
\text { approach ipsilateral }\end{array}$ & & \\
\hline $\begin{array}{l}\text { Yabe y cols., } \\
2014^{18}\end{array}$ & Serie de casos & $\begin{array}{l}\text { Técnica basada en la manipulación para reducción } \\
\text { discal }\end{array}$ & $\mathrm{n}=15$ & \\
\hline Terai, $2014^{19}$ & Serie de casos & Técnica con una mano & $\mathrm{n}=32$ & \\
\hline $\begin{array}{l}\text { Gonai y cols., } \\
2015^{20}\end{array}$ & Serie de casos & Técnica GONAI & $\mathrm{n}=5$ & $80 \%(n=4)$ \\
\hline Heidari, $2015^{21}$ & Reporte de caso & “Técnica semisentada” (posición supina) & $\mathrm{n}=1$ & \\
\hline $\begin{array}{l}\text { Gorchynski y } \\
\text { cols., } 2015^{22}\end{array}$ & Cohorte prospectiva & Técnica de la jeringa & $\mathrm{n}=31$ & $97 \%(n=30)$ \\
\hline $\begin{array}{l}\text { Chhabra y cols., } \\
2015^{23}\end{array}$ & Serie de casos & Reflejo nauseoso & $\mathrm{n}=2$ & \\
\hline $\begin{array}{l}\text { Yeşilołlu y cols., } \\
2015^{24}\end{array}$ & Serie de casos & Técnica de la palanca & $\mathrm{n}=29$ & \\
\hline $\begin{array}{l}\text { Sharma y cols., } \\
2015^{25}\end{array}$ & Revisión narrativa & $\begin{array}{l}\text { Convencional, "convencional modificada" (posición } \\
\text { supina), reflejo nauseoso }\end{array}$ & & \\
\hline $\begin{array}{l}\text { Liddell y cols., } \\
2015^{26}\end{array}$ & Revisión narrativa & Convencional, método extraoral, reflejo nauseoso & & \\
\hline Forshaw, $2015^{8}$ & Revisión narrativa & Convencional, pivote de muñeca, manos unidas & & \\
\hline \multirow{2}{*}{ Xu y cols., $2016^{27}$} & \multirow{2}{*}{$\begin{array}{l}\text { Ensayo clínico } \\
\text { aleatorizado }\end{array}$} & Convencional & $\mathrm{n}=20$ & \\
\hline & & Posición supina & $\mathrm{n}=20$ & \\
\hline \multirow{3}{*}{$\begin{array}{l}\text { Ardehali y cols., } \\
2016^{28}\end{array}$} & \multirow{3}{*}{$\begin{array}{l}\text { Ensayo clínico } \\
\text { aleatorizado }\end{array}$} & Método extraoral & $\mathrm{n}=30$ & $66,7 \%$ \\
\hline & & Método convencional & $\mathrm{n}=30$ & $86,7 \%$ \\
\hline & & Pivote de muñeca & $\mathrm{n}=30$ & $96,7 \%$ \\
\hline $\begin{array}{l}\text { White y cols., } \\
2016^{29}\end{array}$ & Revisión narrativa & Convencional, pivote de muñeca, reflejo nauseoso & & \\
\hline $\begin{array}{l}\text { Lum y Poh, } \\
2017^{30}\end{array}$ & Reporte de caso & Pivote de muñeca & $\mathrm{n}=1$ & \\
\hline $\begin{array}{l}\text { Thomaidis y } \\
\text { cols., } 2018^{9}\end{array}$ & Reporte de caso & Convencional & $\mathrm{n}=1$ & \\
\hline $\begin{array}{l}\text { Prechel y cols., } \\
2018^{4}\end{array}$ & Guía clínica & Convencional, pivote de muñeca, extraoral & & \\
\hline $\begin{array}{l}\text { Stolbizer y cols., } \\
2020^{31}\end{array}$ & $\begin{array}{l}\text { Cohorte } \\
\text { retrospectiva }\end{array}$ & Maniobra de presión sostenida & $\mathrm{n}=42$ & \\
\hline
\end{tabular}


Las técnicas extraorales surgen de la preocupación por el riesgo de mordida incidental y buscan evitar la introducción de las manos del operador en la boca del paciente. Chen y cols. ${ }^{3}$ describieron una técnica de reducción extraoral con el paciente en posición sentada o supina. El operador debe sentarse o pararse frente al paciente y apoyar a un lado el pulgar sobre la eminencia malar y los dedos a nivel del ángulo mandibular, al otro lado el pulgar se apoya sobre la coronoides y los dedos sobre la apófisis mastoides, generando un movimiento de rotación que trata de llevar el ángulo mandibular de un lado hacia anterior al mismo tiempo que el pulgar empuja la coronoides contralateral hacia atrás (Figura 3). Esto genera la reducción de un lado y la consiguiente reducción espontánea del lado contrario.

Gorchynski y cols..$^{22}$ realizaron un estudio prospectivo para evaluar la efectividad de la "técnica de la jeringa". Esta técnica requiere que se coloque una jeringa de 5 o $10 \mathrm{ml}$ entre los molares posteriores unilateralmente; luego el paciente debe realizar movimientos de protrusión y retrusión, a medida que los dientes se deslizan sobre la jeringa el cóndilo es reposicionado a su posición anatómica normal. La ventaja de esta técnica es que no requiere manipulación del operador, por lo que no se aplican fuerzas externas, es cómoda para el paciente y no existe riesgo de mordedura.

En la "técnica de palanca" descrita por Yeşiloğlu²4, con el paciente en posición sentada, se interpone una gasa de aproximadamente $3 \mathrm{~cm}$ de diámetro sobre la zona molar posterior afectada y el operador impulsa el mentón hacia cefálico (Figura 4). En casos bilaterales, se insertan gasas bilaterales y se realiza la misma maniobra.

El 2006, Shun y cols. ${ }^{13}$ describieron el "abordaje ipsilateral”, que manipula solo un lado de la mandíbula a la vez y combina maniobra extraoral, intraoral y combinada para lograr una reducción cerrada. El método extraoral consiste en aplicar presión con el pulgar hacia abajo sobre el cóndilo dislocado de la mandíbula, justo debajo del arco cigomático del paciente.
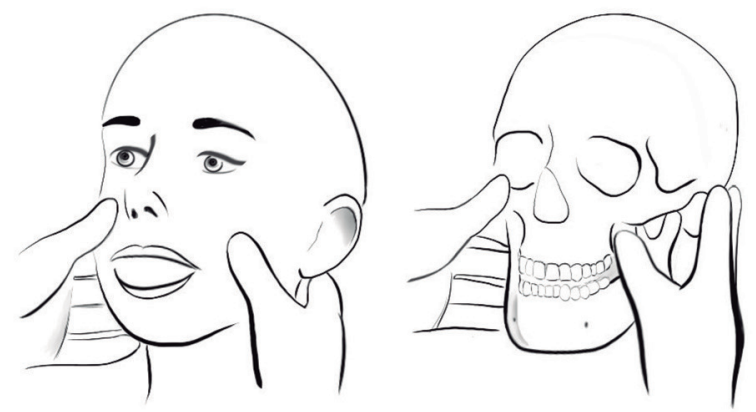

Figura 3. Técnica extraoral: la mano izquierda del operador apoya el dedo pulgar en el malar y dedos índice y medio en el borde posterior de la rama; la mano derecha apoya dedos índice y medio en la mastoides y pulgar en el borde anterior de la rama. Se rota la mandíbula empujando la rama derecha del paciente desde el borde posterior hacia anterior y presionando simultáneamente el borde anterior de la rama izquierda hacia posterior. Se repite la maniobra en el sentido contrario para completar la reducción.

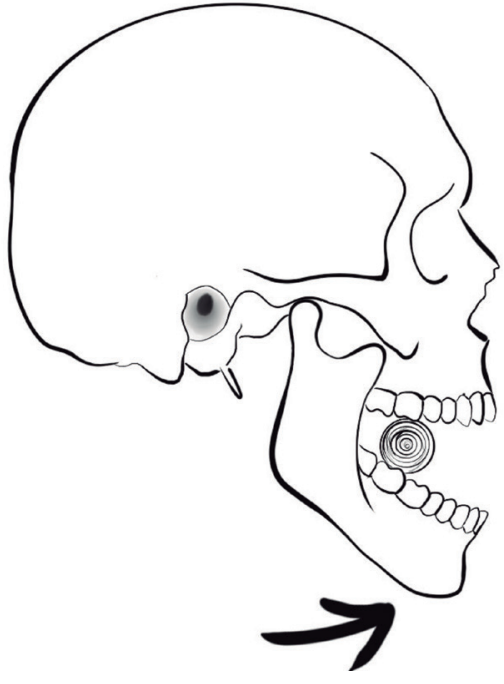

Figura 4. Técnica de palanca: se requiere de una gasa enrollada en forma de tubo, la cual se interpone entre los molares de un lado. A continuación, el operador debe realizar una presión desde el borde inferior del mentón hacia cefálico.

En el método intraoral su pulgar aplica presión hacia abajo sobre la superficie oclusal de los molares inferiores en el lado dislocado del paciente, mientras sostiene la cabeza con la otra mano. En el método combinado, el pulgar aplica presión hacia abajo intraoralmente en un lado y simultáneamente usa el otro pulgar para aplicar presión hacia abajo sobre el cóndilo contralateral extraoralmente.

En los pacientes desdentados totales la efectividad de las técnicas anteriormente descritas está condicionada por la falta de apoyo en el sector oclusal posterior; por esta razón Chhabra y cols. ${ }^{23}$ presentan dos casos clínicos en donde se logra la reducción mediante la activación del reflejo nauseoso al estimular el velo del paladar. Esta técnica fue descrita inicialmente por Awang ${ }^{11}$ en una serie de tres casos.

Terai $^{19}$ describe el uso de una mano para reducir luxaciones uni o bilaterales, con la ventaja adicional de que esta técnica puede ser usada por el paciente para lograr una autorreducción en los casos de luxación recurrente. La maniobra consiste en apoyar el pulgar de la mano contralateral sobre los dientes mandibulares posteriores en el lado afectado, los dedos restantes se colocan bajo del borde inferior de la mandíbula, luego se realiza una rotación con fulcrum en el dedo pulgar mientras los dedos que se encuentran apoyados en el borde mandibular ejercen presión hacia arriba.

En el estudio de Gonai y cols. ${ }^{20}$ la maniobra de reducción extraoral era realizada por el mismo paciente. Una vez que lograban localizar sus cóndilos, un clínico les ordenaba realizar una maniobra de reducción extraoral: empujar el cóndilo hacia adentro, ligeramente hacia atrás y hacia abajo, para reducir la dislocación, mientras que opcionalmente intentan cerrar la boca. Esta técnica es útil para aquellos que no pueden visitar el hospital, es fácil de explicar, realizar y no requiere guantes, gasas o jeringas. 


\section{Comparación de diferentes técnicas}

$\mathrm{Xu}$ y cols. ${ }^{27}$ evaluaron comparativamente la efectividad de la "técnica de posición supina" versus el método convencional para la reducción manual de la luxación aguda no traumática mandibular. El grupo tratado con el método de técnica de posición supina se asoció con una menor duración del procedimiento y menores puntuaciones de dolor durante la maniobra. No se informó de mordida accidental en ninguno de los grupos.

En el 2009, Ardehali y cols. ${ }^{15}$ publicaron un estudio para evaluar la tasa de éxito del método extraoral descrito por Chen ${ }^{3}$ en comparación con el método tradicional. Se intentó reducir la dislocación utilizando el método elegido al azar. La tasa de éxito se calculó para cada método en el primer intento. Como recuperación para los casos fallidos, si el primer método fallaba se intentó el otro método, y si eso tampoco tuvo éxito se administró un relajante muscular (10 mg de diazepam). Las segundas reducciones no se incluyeron en el análisis. De los 29 intentos con el método convencional, 25 tuvieron éxito (86,2\%; IC $95 \%$ : 73-100). Para los otros 4 casos, solo uno pudo reducirse con el nuevo método y los otros tres casos necesitaron un relajante muscular y luego se manejaron con el método convencional. Entre los 29 intentos con el método externo, 16 tuvieron éxito (55,2 \%; IC 95 \%: 39-79) y para otros 13 casos, 10 se redujeron con el método convencional y los otros tres casos recibieron un relajante muscular y luego se redujeron con el nuevo método. Todos los pacientes (38 pacientes con el método convencional y 20 con el método extraoral) podían ser tratados con la maniobra manual y el relajante muscular y ninguno requirió sedación o anestesia general.

Ardehali y cols. ${ }^{28}$, en su ensayo clínico aleatorizado, compararon el método convencional, el extraoral y el pivote de muñeca. Noventa pacientes fueron asignados aleatoriamente a tres grupos. Si el procedimiento de reducción de la mandíbula no tuvo éxito después de dos minutos, se consideró que el método había fallado. Para los clínicos, el método extraoral fue significativamente más difícil de realizar que el convencional $(p=0,003)$ y que el pivote de muñeca $(p=0,000)$. La duración promedio de la reducción para el método extraoral fue de 28,1 segundos y 11,65 segundos para el método de pivote de muñeca. La diferencia entre las tasas de éxito de los tres métodos no fue significativa y el procedimiento extraoral fue significativamente más exitoso en casos unilaterales que en casos bilaterales. El método "pivote de muñeca" puede considerarse como la técnica de preferencia debido a la comodidad para el paciente y la buena tasa de éxito en pacientes que pueden cooperar y en los cuales el riesgo de transmisión de enfermedades infecciosas es bajo. En la situación de riesgo de mordida y luxación unilateral, recomiendan el uso del método extraoral como primera línea, aunque esta técnica es más difícil de realizar para el operador.

\section{Procedimientos de reducción manual con asistencia farmacológica}

Para realizar la reducción manual de las luxaciones mandibulares a menudo se utiliza sedación debido al dolor, la ansiedad y el espasmo de los músculos de la masticación.
En los casos de luxación aguda incluidos por Akinbami y cols. $^{2}, 63$ casos se resolvieron con reducción manual sin ningún tipo de anestesia, 3 casos con reflejo nauseoso, 2 casos se trataron con analgesia intravenosa más sedación y en 14 casos la reducción manual se logró con anestesia general.

El método extraoral de Chen y cols. ${ }^{3}$, la técnica de la jeringa de Gorchynski y cols. ${ }^{22}$, el approach ipsilateral de Shun y cols. ${ }^{13}$ y el pivote de muñeca descrito por Lowery y cols., 20047 criben que no es necesario el uso de analgesia, sedación y/o relajantes musculares para el éxito de la técnica.

Ardehali y cols. ${ }^{28}$ utilizaron el método extraoral, el convencional y el pivote de muñeca y no se requirió relajante muscular para ningún paciente.

La "técnica de palanca" descrita por Yeşiloğlu²4 describe el uso de un relajante muscular (tiocolchicósido $4 \mathrm{mg}$ ) previo a la maniobra y los autores no evaluaron la efectividad de la técnica sin este coadyuvante para la reducción.

Liu ${ }^{32}$ comparó la percepción de dolor y el tiempo de reducción en dos grupos de pacientes con luxaciones mandibulares agudas no traumáticas, uno con la ayuda de óxido nitroso y un grupo control. La percepción de dolor y el tiempo de reducción fueron significativamente menores en el grupo en el que se administró óxido nitroso. Además, se observó una reducción significativa en el tiempo de reducción después de la inhalación de óxido nitroso.

Oliphant y cols. ${ }^{14}$ utilizaron una técnica de reducción convencional bajo sedación con midazolam intravenoso. La sedación consciente se puede utilizar como coadyuvante para todos los métodos de reducción, sin embargo, se recomienda que dos médicos estén presentes y uno sea responsable únicamente de observar al paciente durante la sedación, para evitar complicaciones potencialmente graves de la sedación.

Otra alternativa es el uso de anestésicos locales para el alivio del dolor y la reducción del espasmo muscular reflejo ${ }^{1,5,10,33,34}$. Woodall ${ }^{35}$ describió el uso de anestesia local en la fosa infratemporal para producir relajación de los músculos pterigoideos y así facilitar la reducción, Hebard ${ }^{33}$ describió una infiltración anestésica con técnica extraoral para lograr el mismo objetivo, sin embargo ninguno reportó tasas de éxito ni complicaciones asociadas a la técnica.

Young y cols. ${ }^{36}$ describieron el bloqueo anestésico a los nervios masetérico y temporal profundo, para disminuir el espasmo muscular de los músculos elevadores y el dolor muscular asociado. El bloqueo anestésico se utilizó después de un intento fallido de reducción con la técnica convencional. Posterior a la infiltración, la reducción se realizó rápidamente sin la necesidad de sedación.

\section{DISCUSIÓN}

El objetivo del presente trabajo fue realizar una revisión actualizada sobre los métodos de reducción manual de la luxación mandibular aguda, para luego proponer una secuencia de manejo en el servicio de urgencia, basada en los hallazgos obtenidos.

La luxación mandibular aguda no traumática es poco frecuente, pero es un motivo de consulta constante en los servicios de urgencia. Frente a estos casos, la reducción manual 
rápida y eficaz es clave en disminuir la necesidad de anestesia general, ya que cuanto más tiempo permanezca dislocada la mandíbula, más difícil será el manejo.

En una cohorte retrospectiva ${ }^{37}$ que evaluó el tratamiento en 89 pacientes con luxaciones mandibulares, 49 casos (55\%) se trataron exitosamente con un método manual (con o sin uso de anestesia local y/o sedación) y 40 pacientes requirieron otras maniobras (reducción manual bajo anestesia general en 8 casos, bloqueo intermaxilar en 12 casos o cirugía abierta en 20 pacientes).

No pudimos comparar directamente la eficacia de los métodos manuales, ya que estos se encuentran descritos en reportes o series de casos en los que la técnica fue exitosa. Sin embargo, los ensayos clínicos de Ardehali ${ }^{15,28}$ nos permiten estimar que el método convencional tiene una efectividad cercana al $86 \%$, el método de pivote de muñeca al $96,7 \%$ y que el método extraoral es menos efectivo con un porcentaje de éxito entre 55,2 y $66,7 \%$.

Las limitaciones de esta revisión se deben principalmente a la inclusión de tipos de diseños de estudio diferentes, a la escasa cantidad de ensayos clínicos publicados y a la imposibilidad de analizar y comparar estadísticamente los resultados de los estudios.

El amplio uso de la técnica de reducción manual tradicional es discutible, ya que muchas veces la elección se fundamenta en la experiencia del operador, quien maneja las técnicas que conoció en su formación. Esto queda de manifiesto en la falta de ensayos clínicos que comparen diferentes procedimientos de reducción.

Existe poca rigurosidad en el reporte de las causas de luxación, el tiempo de evolución hasta el momento de consulta, los antecedentes de luxaciones previas y cómo estos factores influyen en el éxito de las técnicas descritas. A pesar de que la información es escasa, los datos descriptivos publicados ${ }^{28}$ indican que no existe una relación entre estos factores antes mencionados y el éxito de las técnicas de reducción manual.

La reducción manual tradicional requiere de aplicación de fuerza por parte del operador, lo que determina que para que el paciente pueda tolerar el procedimiento, comúnmente se asocie la técnica al uso de coadyuvantes, como analgesia intravenosa y sedación consciente ${ }^{22}$. Esto ha llevado al desarrollo de técnicas alternativas para la reducción manual en luxaciones mandibulares agudas no traumáticas. El riesgo de lesión para el operador durante la maniobra es otro factor que se ha intentado minimizar con la descripción de técnicas como la extraoral, de la jeringa, técnica de la palanca y el uso del reflejo nauseoso, sin embargo, pareciera que estas técnicas se asocian a una menor tasa de éxito y a mayor dificultad para el clínico que la realiza. La elección de una técnica sobre otra no se fundamenta en criterios objetivos de éxito clínico.

\section{Propuesta de secuencia de manejo en el servicio de urgencia}

Con base en la revisión presentada, proponemos la siguiente secuencia de tratamiento para luxaciones mandibulares en el servicio de urgencia (Figura 5).

La evaluación imagenológica mediante radiografías convencionales o tomografía computada es perentoria en casos de luxaciones mandibulares asociadas a trauma facial. En los casos de luxación espontánea la confirmación del diagnóstico clínico mediante imágenes tiene importancia médico-legal y

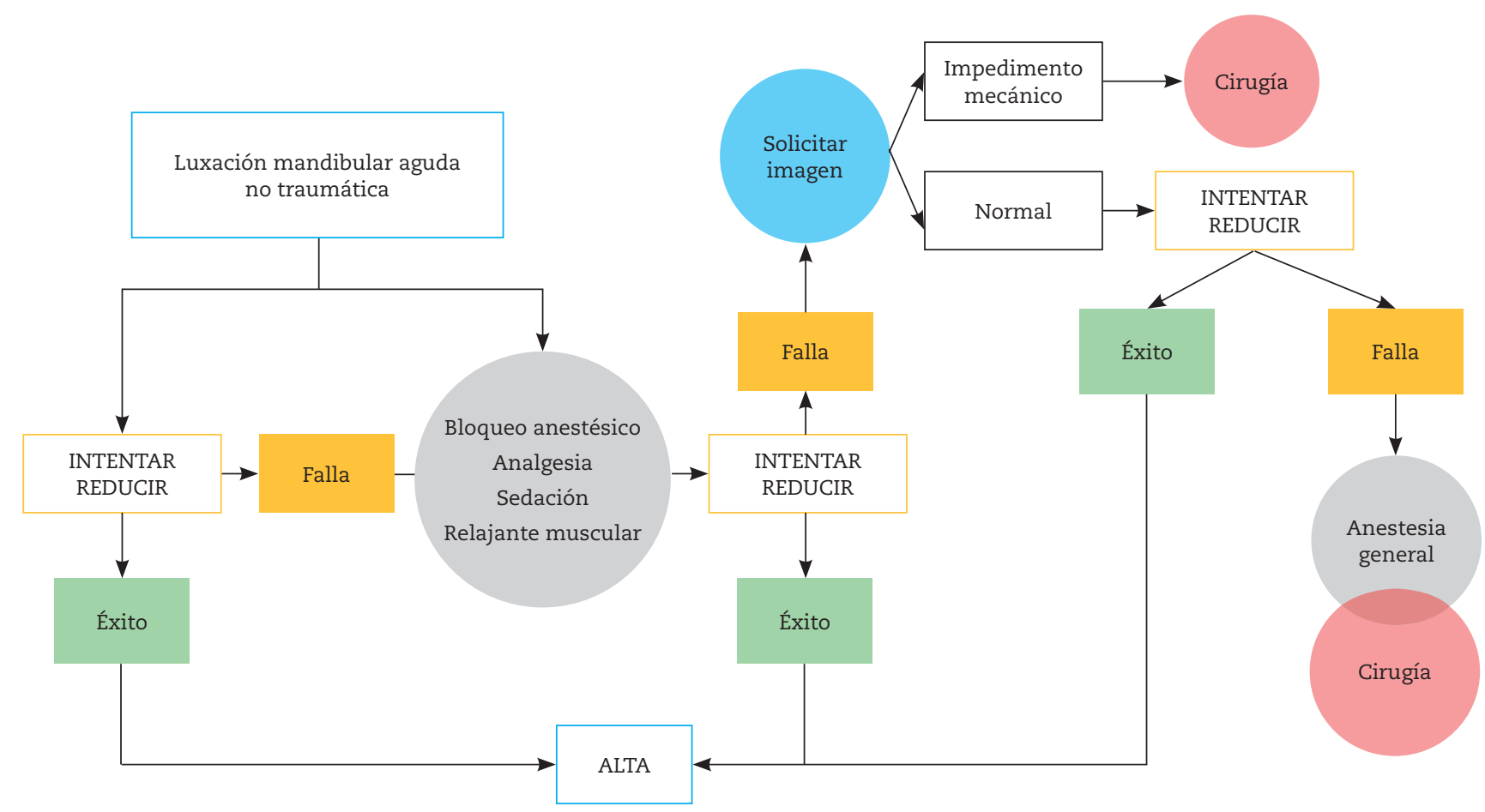

Figura 5. Secuencia de tratamiento. 
previene la omisión de un diagnóstico, sin embargo no lo consideramos de primera línea debido a que la toma de imágenes no está siempre disponible en servicios de atención primaria y no debe retrasar las maniobras de reducción.

No existe un método que sea probadamente superior al resto y la elección de la técnica de reducción dependerá de la experiencia y comodidad del operador. Para esto proponemos iniciar con algún método que evite introducir los dedos en la boca del paciente, entre las alternativas se encuentran el método extraoral, la técnica de la jeringa y la técnica de palanca. En pacientes con luxación bilateral, el método extraoral tiene una baja tasa de éxito $(54,5 \%$ versus $96,7 \%$ en pacientes con luxación unilateral) y, por lo tanto, su uso solo se recomienda en caso de un aumento de riesgo de mordedura o infección (por ejemplo, en pacientes con demencia, pacientes con hepatitis $\mathrm{C})^{4,15}$.

Si estas opciones no son efectivas, un método cómodo para el clínico y que tiene una buena tasa de efectividad sería el de pivote de muñeca, seguido por el método de posición supina y finalmente por el método convencional. Este último, a pesar de ser muy efectivo, se asocia a dificultad para el operador y dolor para el paciente durante la maniobra.

Luego del alta las indicaciones incluyen el reposo de la articulación, con dieta blanda por cinco a siete días y el uso de antinflamatorios los primeros días. Se sugiere complementar la atención de urgencia con evaluación por un especialista en cirugía maxilofacial si el episodio se encuentra asociado a un cuadro de luxaciones recurrentes (inclusive si el paciente logra autorreducción en la mayoría de los casos), ya que puede ser necesario complementar con un estudio morfológico de la articulación.

En casos en donde después de la reducción manual el paciente vuelve a luxarse inmediatamente, es útil el uso de un vendaje de Barton para mantener la mandíbula en posición. Otra alternativa es el uso de un collar cervical blando, que logra el mismo objetivo en forma fácil y efectiva ${ }^{23,38}$.

Es fundamental para los clínicos conocer las diferentes técnicas presentadas, lo que permite utilizarlas en los casos en donde otras técnicas fracasan, minimizando el uso de coadyuvantes u otras maniobras más complejas, como bloqueos regionales o anestesia general.

Faltan estudios que comparen distintas técnicas con un operador entrenado considerando variables tanto del paciente, como de la reducción y la comodidad para el operador. La mejor técnica de reducción manual es aquella que entrega mayor comodidad al profesional, produce menor dolor en el paciente y se asocia a una mayor tasa de éxito.

\section{CONFLICTOS DE INTERESES}

Los autores declaran que no hubo conflicto de intereses en la elaboración de este estudio y que no existen fuentes de financiación públicas o privadas en la realización del presente trabajo.

\section{B I B L I O G R A F Í A}

1. Chan TC, Harrigan RA, Ufberg J, Vilke GM. Mandibular Reduction. J Emerg Med. 2008;34(4):435-40. DOI: 10.1016/j.jemermed.2007.06.037.
2. Akinbami BO. Evaluation of the mechanism and principles of management of temporomandibular joint dislocation. Systematic review of literature and a proposed new classification of temporomandibular joint dislocation. Head Face Med. 2011;7:10. DOI: 10.1186/1746-160X-7-10.

3. Chen YC, Chen CT, Lin CH, Chen YR. A safe and effective way for reduction of temporomandibular joint dislocation. Ann Plast Surg. 2007;58(1):105-8. DOI: 10.1097/01.sap.0000232981.40497.32.

4. Prechel U, Ottl P, Ahlers OM, Neff A. The treatment of temporomandibular joint dislocation - A systematic review. Dtsch Arztebl Int. 2018;115(5):59-64. DOI: 10.3238/arztebl.2018.0059.

5. Hillam J, Isom B. Mandible Dislocation. Treasure Island (FL): StatPearls Publishing; 2020.

6. Abrahamsson H, Eriksson L, Abrahamsson P, Häggman-Henrikson $\mathrm{B}$. Treatment of temporomandibular joint luxation: a systematic literature review. Clin Oral Investig. 2020;24(1):6170. DOI: 10.1007/s00784-019-03126-1.

7. Lowery LE, Beeson MS, Lum KK. The wrist pivot method, a novel technique for temporomandibular joint reduction. J Emerg Med. 2004;27(2):167-70. DOI: 10.1016/j.jemermed.2004.03.007

8. Forshaw RJ. Reduction of temporomandibular joint dislocation: An ancient technique that has stood the test of time. $\mathrm{Br}$ Dent J. 2015;218(12):691-3. DOI: 10.1038/sj.bdj.2015.438.

9. Thomaidis V, Tsoucalas G, Fiska A. The Hippocratic Method for the Reduction of the Mandibular Dislocation, an Ancient Greek Procedure Still in Use in Maxillofacial Surgery. Acta Med Acad. 2018;47(1):139-43. DOI: 10.5644/ama2006-124.224.

10. Stakesby Lewisg JE. A simple technique for reduction of longstanding dislocation of the mandible. Br J Oral Surg. 1981;19(1): 52-6. DOI: 10.1016/0007-117X(81)90021-4.

11. Awang MN. A new approach to the reduction of acute dislocation of the temporomandibular joint: A report of three cases. Br J Oral Maxillofac Surg. 1987;25(3):244-9. DOI: 10.1016/S02664356(87)80025-6.

12. Luyk NH, Larsen PE. The diagnosis and treatment of the dislocated mandible. Am J Emerg Med. 1989;7(3):329-35. DOI: 10.1016/0735-6757(89)90181-2.

13. Shun TAT, Wai WT, Chiu LC. A case series of closed reduction for acute temporomandibular joint dislocation by a new approach. Eur J Emerg Med. 2006;13(2):72-5. DOI: 10.1097/01. mej.0000192046.19977.5a.

14. Oliphant R, Key B, Chung D, Dawson C. Bilateral temporomandibular joint dislocation following pulmonary function testing: A case report and review of closed reduction techniques. Emerg Med J. 2008;25(7):435-6. DOI: 10.1136/emj.2007.055038.

15. Ardehali MM, Kouhi A, Meighani A, Rad FM, Emami H. Temporomandibular joint dislocation reduction technique: A new external method vs. the traditional. Ann Plast Surg. 2009;63(2): 176-8. DOI: 10.1097/SAP.0b013e31818937aa.

16. Cheng D. Unified Hands Technique for Mandibular Dislocation. J Emerg Med. 2010;38(3):366-7. DOI: 10.1016/j.jemermed.2008.12.022.

17. Osawa Gutierrez LM, Grossmann TK, Grossmann E. Anterior dislocation of head of mandible : diagnosis and treatment. Rev Dor São Paulo. 2011;12(1):64-70. DOI: 10.1590/S180600132011000100014.

18. Yabe T, Tsuda T, Hirose S, Ozawa T, Kawai K. Treatment of acute temporomandibular joint dislocation using manipulation technique for disk displacement. J Craniofac Surg 2014;25(2):596-7. DOI: 10.1097/SCS.0000000000000676.

19. Terai H, Kasuya S, Nakagawa Y, Ueno T. The use of only one hand for the reduction of a temporomandibular joint dislocation: A technique suitable for self-reduction. Int J Oral Maxillofac Surg. 2014;43(5):663-4. DOI: 10.1016/j.ijom.2013.11.008.

20. Gonai S, Doi K, Noguchi K, Kanao K, Gommori S, Saito Y, et al. Extraoral autoreduction of temporomandibular joint dislocation: A preliminary clinical study. Am J Emerg Med. 2015;33(4):588-9. DOI: 10.1016/j.ajem.2015.01.011. 
21. Heidari SF. The new technique for reduction of bilateral mandibular dislocation. Am J Emerg Med. 2015;33(9):6757. DOI: 10.1016/j.ajem.2015.02.042.

22. Gorchynski JA, Karabidian E, Sanchez M. The "syringe" technique: A hands-free approach for the reduction of acute nontraumatic temporomandibular dislocations in the emergency department. J Emerg Med. 2014;47(6):676-81. DOI: 10.1016/j. jemermed.2014.06.050.

23. Chhabra S, Chhabra N, Gupta P. Recurrent Mandibular Dislocation in Geriatric Patients: Treatment and Prevention by a Simple and Non-invasive Technique. J Maxillofac Oral Surg 2015;14(Suppl. 1):231-4. DOI: 10.1007/s12663-012-0454-7.

24. Yeşilołlu N, Sarici M, Şirinołlu H, Temiz G, Güvercin E, Filinte GT. The lever technique for the external reduction of temporomandibular joint dislocation. J Plast Reconstr Aesthetic Surg. 2015;68(1):123-5. DOI: 10.1016/j.bjps.2014.08.052.

25. Sharma N, Singh A, Pandey A, Verma V, Singh S. Temporomandibular joint dislocation. Natl J Maxillofac Surg. 2015;6(1):16. DOI: 10.4103/0975-5950.168212.

26. Liddell A, Perez DE. Temporomandibular Joint Dislocation. Oral Maxillofac Surg Clin North Am. 2015;27(1):125-36. DOI: 10.1016/j.coms.2014.09.009.

27. Xu J, Dong S, Zhou H, Somar M, Lv K, Li Z. The supine position technique method is better than the conventional method for manual reduction of acute nontraumatic temporomandibular joint dislocation. J Craniofac Surg. 2016;27(4):919-22. DOI: 10.1097/SCS.0000000000002645.

28. Ardehali MM, Tari N, Bastaninejad S, Amirizad A. Comparison of different approaches to the reduction of anterior temporomandibular joint dislocation : a randomized clinical trial. Int J Oral Maxillofac Surg. 2016;45(8):1009-14. DOI: 10.1016/j. ijom.2016.04.015.
29. White T, Hedderick V, Ramponi DR. Dislocation of the Temporomandibular Joint and Relocation Procedures. Adv Emerg Nurs J. 2016;38(3):177-82. DOI: 10.1097/TME.0000000000000110.

30. Lum V, Poh J. Refractory Temporomandibular Joint Dislocation - Reduction Using the Wrist Pivot Method. Clin Pract Cases Emerg Med 2017;1(4):380-3. DOI: 10.5811/cpcem.2017.9.35834.

31. Stolbizer F, Saiegh J, Andrada MM. Anterior dislocation of the temporomandibular joint: a simplified non-traumatic manual technique. J Man Manip Ther. 2020;28(4):246-50. DOI: 10.1080/10669817.2019.1704516.

32. Liu M, Lv K. Clinical Trial of Manual Reduction of Temporomandibular Joint Dislocation after Inhalation of Nitrous Oxide. J Craniofac Surg. 2019;30(8):2549-50. DOI: 10.1097/SCS.0000000000005816.

33. Hebard RL. ITMJ reduction. Otolaryngol Head Neck Surg. 2014;151(6):1086-7. DOI: 10.1177/0194599814544888

34. Hsiung CP, Suresh S. Closed reduction of a temporomandibular joint dislocation: Is this possible with regional anesthesia? Paediatr Anaesth. 2008;18(12):1269-70. DOI: 10.1111/j.1460-9592.2008.02790.x.

35. Woodall CE, Padaki P, Siddiqui A, Bayoumi S. The use of intraoral local anaesthetic to aid reduction of acute temporomandibular joint dislocation. J Stomatol Oral Maxillofac Surg. 2019;120(2):152-3. DOI: 10.1016/j.jormas.2018.10.013.

36. Young AL, Khan J, Thomas DC, Quek SYP. Use of masseteric and deep temporal nerve blocks for reduction of mandibular dislocation. Anesth Prog. 2009;56(1):9-13. DOI: 10.2344/0003-3006-56.1.9.

37. Ugboko VI, Oginni FO, Ajike SO, Olasoji HO, Adebayo ETA. A survey of temporomandibular joint dislocation : aetiology , demographics, risk factors and management in 96 Nigerian cases. Int J Oral Maxillofac Surg. 2005;34(5):499-502. DOI: 10.1016/j.ijom.2004.10.025.

38. Jaisani MR, Pradhan L, Sagtani A. Use of Cervical Collar in Temporomandibular Dislocation. J Maxillofac Oral Surg. 2015;14(2):470-1. DOI: 10.1007/s12663-013-0505-8. 